\title{
Retinopatía esclopetaria: reporte de caso y revisión de literatura
}

\section{Retinitis sclopetaria: Case report and literature review}

\author{
Ma. Paula Dussán-Vargas ${ }^{1 *}$, Alejandro Londoño ${ }^{1}$, Laura E. León-Pachón ${ }^{1}$, Manuela Franco-Sánchez ${ }^{1}$, \\ Ma. Camila Escobar-Arias ${ }^{2}$ y Andrés Amaya-Espinosa ${ }^{3}$ \\ ${ }^{1}$ Departamento de Oftalmología, Universidad de la Sabana, Cundinamarca; ${ }^{2}$ Facultad de Medicina, Universidad de los Andes, Bogotá; ${ }^{3}$ Especialidad \\ en Retina y Vitreo, Departamento de Oftalmología, Universidad de la Sabana, Cundinamarca. Colombia
}

\section{Resumen}

Introducción: La retinopatía esclopetaria se presenta en casos de trauma ocular cerrado por proyectiles de alta velocidad a nivel orbitario y periorbitario con alteraciones secundarias en la coroides, retina, epitelio pigmentario de la retina y membrana de Bruch. Por la rara ocurrencia de este mecanismo de trauma, es una complicación poco frecuente del trauma ocular cerrado que se presenta en el momento inicial del trauma y genera secuelas a largo plazo. Objetivo: Dar a conocer las características clínicas y las secuelas de la retinopatía esclopetaria. Diseño del estudio: Observacional. Material y métodos: Se presenta el caso clínico de un hombre de 45 años con antecedente de herida por arma de fuego hace 27 años, con restos de esquirlas metálicas a nivel frontal, con disminución de agudeza visual crónica, hallazgos de catarata subcapsular posterior y a la fundoscopia cicatriz coriorretinal compatible con retinopatía esclopetaria. Se indica manejo médico con observación de la alteración del segmento posterior y manejo quirúrgico de la catarata secundaria al trauma. Conclusiones: La retinopatía esclopetaria puede generar daños agudos y secuelas que conlleven disminución de la agudeza visual; el manejo es médico observacional. Según la localización de los hallazgos se definirán las secuelas visuales. El diagnóstico oportuno y seguimiento son el pilar del tratamiento.

Palabras clave: Esclopetaria. Coriorretinitis esclopetaria traumática. Ruptura coriorretinal traumática. Trauma ocular.

\begin{abstract}
Background: Retinitis sclopetaria occurs in cases of closed ocular trauma caused by high-speed bullets at the orbital and periorbital tissues with secondary alterations in the choroid, retina, RPE and Bruch's membrane. Due to the rare occurrence of this mechanism, it is a rare complication of closed ocular trauma that occurs at the initial moment of trauma and generates long-term sequelae. Objective: To present clinical characteristics and the sequelae of a case of retinitis sclopetaria. Study design: Observational Material and methods: We present the clinical case of a 45-year-old man with a history of firearm injury 27 years ago, with remains of metallic splinters at the frontal level, with decreased chronic visual acuity, findings of a posterior subcapsular cataract and fundoscopy of chorioretinal scar compatible with retinitis sclopetaria. Medical management was indicated with observation and surgical management of the cataract secondary to trauma. Conclusions: Retinitis sclopetaria can cause acute damage and sequelae that lead to decreased visual acuity; treatment is observation. Visual sequelae is defined depending on the location of the retinal alterations. Diagnosis and follow-up are the mainstay of management.
\end{abstract}

Key words: Sclopetaria. Traumatic chorioretinitis sclopetaria. Traumatic chorioretinal rupture. Ocular trauma.

Correspondencia:

*Ma. Paula Dussán-Vargas

E-mail: mariapauladussan.mpdv@gmail.com

$0120-0453 / 02021$

CC BY-NC-ND (http://creativecommons.org/licenses/by-nc-nd/4.0/).
Fecha de recepción: 05-02-2021

Fecha de aceptación: 30-05-2021

DOI: 10.24875/RSCO.M21000012
Disponible en internet: 28-12-2021 Rev Soc Colomb Oftalmol. 2021;54(2):92-95 www. revistaSCO.com 


\section{Introducción}

La retinopatía esclopetaria es una condición poco frecuente que se puede presentar en las lesiones por proyectiles de alta velocidad que pasan adyacentes a la cavidad orbitaria sin penetrar el globo ocular, generando mecanismos de trauma contundente a nivel intraconal que generan fuerzas de aceleración-desaceleración intraoculares ${ }^{1,2}$. Se relaciona con rupturas coroideas y retinianas que dejan expuesta la esclera y generan cicatrices fibrosas adherentes a este nivel ${ }^{3}$. A continuación, presentamos un caso de retinopatía esclopetaria relacionada con herida por arma de fuego.

\section{Caso clínico}

Paciente de sexo masculino de 45 años de edad, quien ingresa al servicio de urgencias por cuadro clínico de trauma craneoencefálico leve en región frontal con objeto contundente (ladrillo) en contexto de riña callejera. Refiere antecedente de importancia de herida por arma de fuego en región malar derecha hace 27 años y disminución de agudeza visual crónica. Presenta herida en región frontal, con tomografía computarizada (TC) simple de cráneo que evidencia fractura frontal derecha ligeramente deprimida y múltiples restos de material metálico compatible con esquirlas de proyectil de arma de fuego a nivel malar derecho (Fig. 1). La fractura fue intervenida quirúrgicamente por neurocirugía.

Por referir visión borrosa monocular derecha de larga data, es valorado por oftalmología. Al examen físico oftalmológico se encuentra en ojo derecho: edema palpebral superior moderado asociado a equimosis, con agudeza visual 20/100 y corrección con agujero estenopeico a 20/80; se evidencia catarata subcapsular posterior y secuelas de coriorretinopatía esclopetaria dada por cicatriz coriorretiniana fibrosa y blanquecina de 1 diámetro de disco al final de la arcada vascular inferotemporal (Fig. 2). En el ojo izquierdo el examen oftalmológico se encuentra dentro de límites normales.

Se solicita TC de órbitas, la cual no reporta alteraciones orbitarias, con único hallazgo de restos de material metálico descrito en TC de cráneo previo (Fig. 3). Es valorado por retinología, donde se considera que el paciente requiere manejo quirúrgico de catarata en ojo derecho y que los hallazgos en segmento posterior de este corresponden a secuelas del trauma antiguo por arma de fuego previamente mencionado, sin indicación actual de manejo adicional.

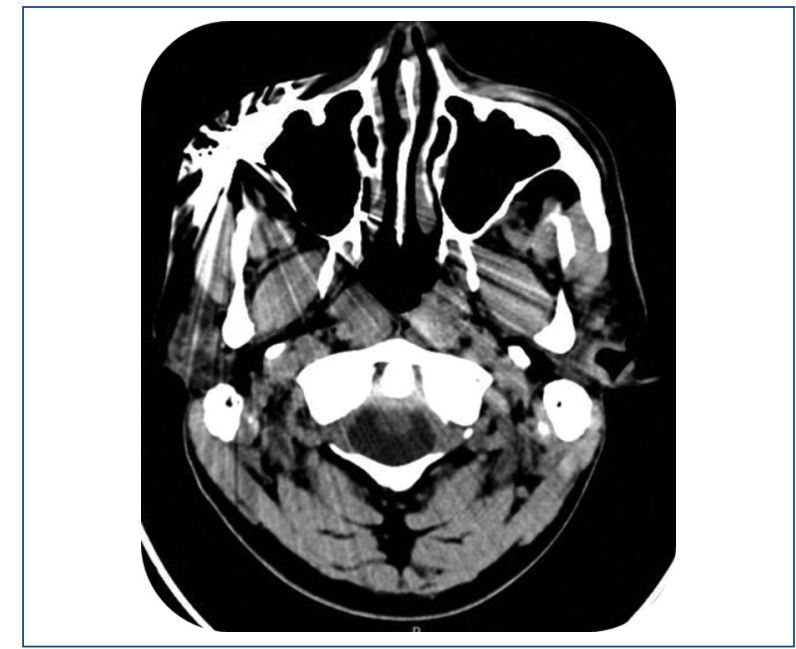

Figura 1. Tomografía axial computarizada de cráneo simple, corte axial con evidencia de restos de esquirlas metálicas a nivel malar derecho.

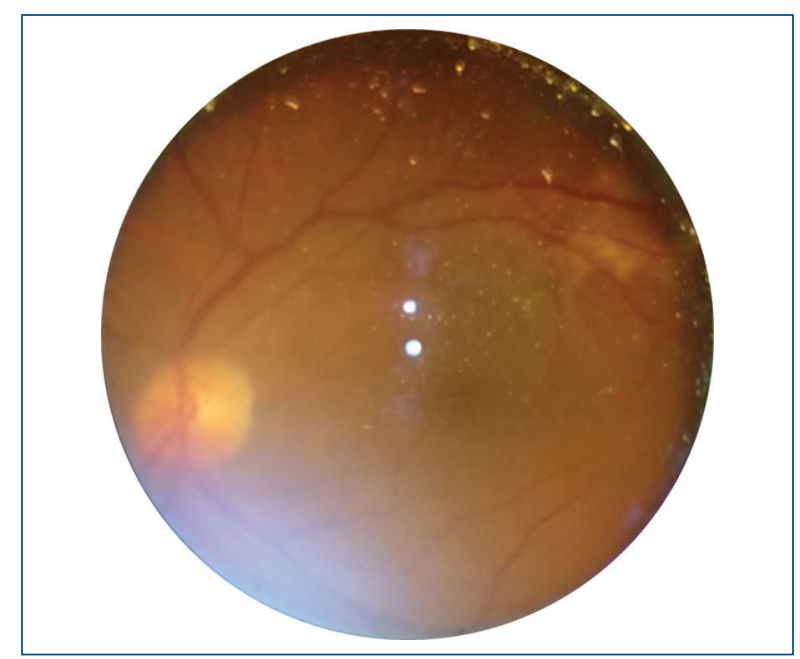

Figura 2. Bajo dilatación farmacológica autorizada con visualización con lente de $20 \mathrm{D}$ se evidencian medios levemente opacos, cicatriz coriorretiniana fibrosa blanquecina de 1 diámetro de disco al final de la arcada vascular inferotemporal.

\section{Discusión}

La coriorretinitis esclopetaria es una de las posibles complicaciones derivadas del trauma ocular cerrado con compromiso del segmento posterior. Fue descrita por primera vez en 1901 por Goldzieher, quien relacionó los traumas oculares cerrados por heridas por arma de fuego a nivel periorbitario u orbitario sin contacto directo con el globo ocular con la ruptura completa de la coroides y la retina ${ }^{1}$. Se considera que el 


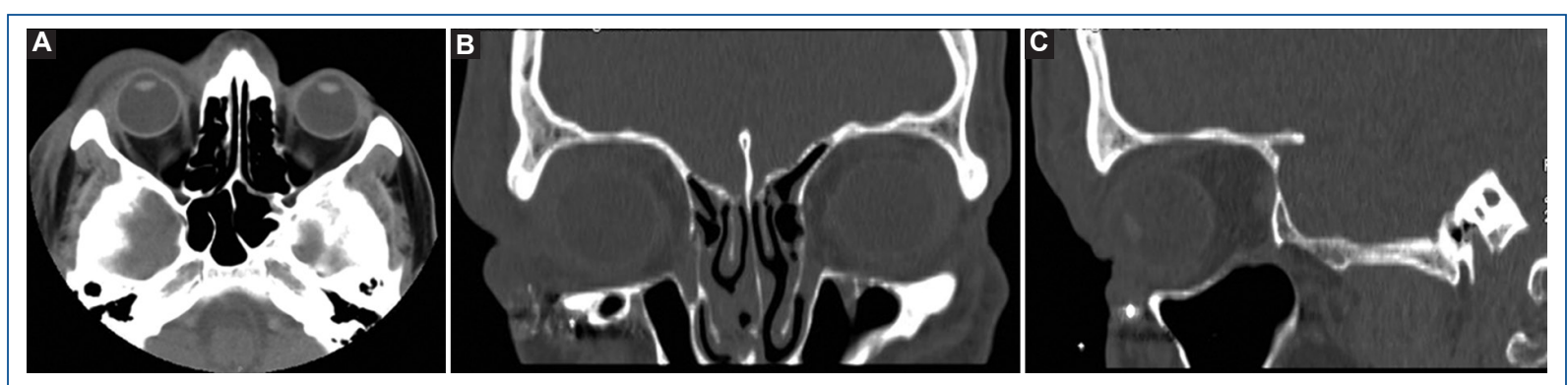

Figura 3. Tomografía axial computarizada de órbitas. A: corte axial. B: corte coronal. C: corte sagital. Con cavidades orbitarias íntegras, sin compromiso de grasa intraconal ni extraconal, con globos oculares tónicos, sin evidencia de ruptura ocular, con edema de tejidos blandos palpebrales derechos.

término tiene dos posibles orígenes: la palabra en latín sclopetum, que se refiere a un tipo de arma italiana usada en el siglo XIV o de la palabra inglesa sclow, que significa rasguño o desgarro en español ${ }^{2-4}$. Años después fue cambiando el nombre a retinopatía esclopetaria, una vez fue entendida su etiología y dado que se describió que los hallazgos encontrados en la fundoscopia corresponden a una cicatriz y no a una inflamación coriorretiniana'.

La retinopatía esclopetaria es una manifestación rara de trauma ocular de la cual los datos epidemiológicos son escasos, siendo los dos estudios más grandes de ocho casos, publicado en 1994 por Martin, et al., y de 13 casos, publicado por Ahmadabadi, et al. en 20095,6. En el estudio de Martin, et al. todos los pacientes eran hombres con edad promedio de 19 años y en el estudio de Ahmadabadi, et al. 11 de 13 pacientes eran hombres con edad promedio de $16 \pm 6$ años, por lo que la retinopatía esclopetaria parece ser un problema relacionado con el sexo. Se ha visto que ocurre más en hombres y jóvenes que en otras lesiones oculares por trauma, principalmente en países con conflicto armado, sin tener una incidencia $y / 0$ prevalencia exacta ${ }^{3,5,6}$. Dada la poca información epidemiológica sobre esta patología consideramos importante la exposición de casos que contribuyan a la realización de estudios de caracterización de la retinopatía esclopetaria.

La etiología principal son las balas de arma de fuego, sin embargo, cualquier tipo de proyectil de alta de velocidad que genere impacto en los tejidos orbitarios o periorbitarios puede ocasionar esta patología 7 .

La fisiopatología propuesta tiene como principal causa la deformación rápida del globo ocular, dada por las fuerzas de aceleración-desaceleración por los proyectiles de alta velocidad; esto genera estrés a nivel de la esclera, retina, coroides y vítreo, por lo que se presenta una ruptura en la coroides y la retina; la esclera y el vítreo permanecen intactos por su mayor capacidad tensil intrínseca ${ }^{1,3}$. También ha sido descrita una segunda hipótesis relacionada con lesiones indirectas provocadas por la transmisión de las fuerzas a través de la cavidad ósea orbitaria, lo cual puede conllevar a lesiones remotas o contralaterales al sitio de impacto del proyectil ${ }^{2,5}$.

Histopatológicamente han sido descritos defectos maculares de la membrana de Bruch y la coroides, con pérdida de fotorreceptores e hiperplasia del epitelio pigmentario de la retina (EPR). La retina y la coroides son reemplazadas por tejido conectivo denso y generan cicatrices fibrosas adherentes en las áreas de ruptura 4 .

Los hallazgos oftalmológicos pueden variar dependiendo del mecanismo del trauma y la velocidad del proyectil; se debe diferenciar de patologías como commotio retinae, ruptura coroidea y agujeros maculares traumáticos ${ }^{5,8}$. Se diferencia la retinopatía esclopetaria por el extenso daño que compromete la coroides, membrana de Bruch, EPR y retina neurosensorial, siendo el hallazgo típico una cicatriz blanquecina en forma de membrana en el polo posterior, con bordes irregulares que presentan dispersión y/o acumulación de pigmento ${ }^{4,9}$.

El diagnóstico es clínico, basado en la anamnesis, el tipo de trauma y el examen oftalmológico, principalmente en la fundoscopia ${ }^{8}$. Es de vital importancia precisar si el compromiso de la agudeza visual se presentó súbitamente relacionado con el trauma o si es una patología crónica como en el caso descrito. Puede ser útil la ecografía ocular, que confirma adelgazamiento del tejido coriorretiniano con ruptura de este, sin evidencia de separación ni desprendimiento de la esclera ${ }^{3}$. También se recomienda la realización de TC de órbitas 
para descartar fracturas o compromiso adicional orbitario dado el mecanismo de trauma ${ }^{10}$.

Se considera que presenta un bajo riesgo de desprendimiento de retina teniendo en cuenta que las zonas de ruptura tienen retracción a nivel retinal y coroideo como una unidad, por lo cual disminuye la posibilidad de acumulación de líquido subretiniano; asociado a esto, la edad de presentación es en personas jóvenes, por lo cual el vítreo no presenta alteraciones predisponentes para desprendimiento de retina. Además, el vítreo está intacto en la zona de lesión coriorretinal y la proliferación de tejido conectivo genera una unión firme y adherente entre retina, coroides y esclera ${ }^{1-3}$.

El tratamiento de elección en estos casos es la observación, con monitoreo de la agudeza visual; raramente se requiere manejo quirúrgico para reparación de desprendimiento de retina ${ }^{2,3,6}$. El pronóstico depende del estado de la mácula y la ubicación de la lesión, con peores resultados en los casos de lesiones con compromiso macular. También se ha descrito una posible relación entre las lesiones extramaculares ubicadas a nivel superior y temporal, con peores resultados visuales ${ }^{4,10}$.

En el caso presentado se evidencia la relación de las alteraciones coriorretinianas con el antiguo trauma ocular cerrado por proyectil de arma de fuego; se considera que en este caso la agudeza visual está parcialmente comprometida por la localización de la lesión extramacular, sin requerimiento de manejo adicional a la observación y seguimiento clínico.

\section{Conclusión}

La retinopatía esclopetaria es una complicación poco frecuente del trauma ocular cerrado, que genera alteraciones en el segmento posterior del globo ocular. Su principal secuela es la disminución de la agudeza visual, la cual estará comprometida según la ubicación de la lesión. El manejo de elección es la observación según sea el caso de cada paciente; podemos concluir que en el caso presentado las características del trauma asociado a la localización de la lesión permitieron un mejor pronóstico visual, por lo cual lo más importante en un evento de trauma ocular es la individualización de cada paciente, teniendo en cuenta todas las posibles lesiones que se pueden presentar derivadas de un trauma y sus secuelas potenciales, sin tener que presentarse necesariamente como un trauma ocular abierto.

\section{Financiamiento}

Los autores no recibieron patrocinio para llevar a cabo este artículo.

\section{Conflicto de intereses}

Los autores declaran no tener conflicto de intereses.

\section{Responsabilidades éticas}

Protección de personas y animales. Los autores declaran que para esta investigación no se han realizado experimentos en seres humanos ni en animales.

Confidencialidad de los datos. Los autores declaran que han seguido los protocolos de su centro de trabajo sobre la publicación de datos de pacientes.

Derecho a la privacidad y consentimiento informado. Los autores han obtenido el consentimiento informado de los pacientes y/o sujetos referidos en el artículo. Este documento obra en poder del autor de correspondencia.

\section{Bibliografía}

1. Papakostas TD, Yonekawa Y, Skondra D, Vavvas DG. Traumatic chorioretinal rupture (sclopetaria). Int Ophthalmol Clin. 2013:53(4):119-25.

2. Beatty S, Smyth K, Eong KA, Lavin MJ. Chorioretinitis sclopetaria. Injury. 2000;31(1):55-60.

3. Fraser EJ, Haug SJ, McDonald HR. Clinical presentation of chorioretinitis sclopetaria. Retin Cases Brief Rep. 2014;8(4):257-9.

4. Ludwig CA, Shields RA, Do DV, Moshfeghi DM, Mahajan VB. Traumatic chorioretinitis sclopetaria: Risk factors, management, and prognosis. Am J Ophthalmol Case Rep. 2019;14:39-46.

5. Ahmadabadi MN, Karkhaneh R, Roohipoor R, Tabatabai A, Alimardani A. Clinical presentation and outcome of chorioretinitis sclopetaria: a case series study. Injury. 2010;41(1):82-5.

6. Martin DF, Awh CC, McCuen II BW, Jaffe GJ, Slott JH, Machemer R. Treatment and pathogenesis of traumatic chorioretinal rupture (sclopetaria). Am J Ophthalmol. 1994:117(2):190-200.

7. Georgalas I, Koutsandrea C, Papaconstantinou D, Kampougeris G, Ladas I. Evolution of retinitis sclopetaria after blunt trauma. Clin Exp Ophthalmol. 2009;37(9):896-7.

8. Richards RD, West E, CMeisels AA. Chorioretinitis sclopetaria. Trans Am Ophthalmol Soc. 1968;66:214-32.

9. Ku CA, Jeng F, Reynolds AL, Patel SP. Bilateral ocular injuries with globe perforation and retinitis sclopetaria from birdshot shotgun: A case report and review of the literature. Trauma Case Rep. 2020;28:100329.

10. Blanch RJ, Good PA, Shah P, Bishop JR, Logan A, Scott RA. Visual outcomes after blunt ocular trauma. Ophthalmology. 2013;120(8):1588-91. 